\title{
The Jordan-Hölder theorem with uniqueness for groups and semimodular lattices
}

\author{
GÁbor CzÉdli And E. TAmás SCHMidt
}

\begin{abstract}
For subnormal subgroups $A \triangleleft B$ and $C \triangleleft D$ of a given group $G$, the factor $B / A$ will be called subnormally down-and-up projective to $D / C$, if there are subnormal subgroups $X \triangleleft Y$ such that $A Y=B, A \cap Y=X, C Y=D$ and $C \cap Y=X$. Clearly, $B / A \cong D / C$ in this case. As G. Grätzer and J. B. Nation [6] have just pointed out, the standard proof of the classical Jordan-Hölder theorem yields somewhat more than widely known; namely, the factors of any two given composition series are the same up to subnormal down-and-up projectivity and a permutation. We prove the uniqueness of this permutation.

The main result is the analogous statement for semimodular lattices. Most of the paper belongs to pure lattice theory; the group theoretical part is only a simple reference to a classical theorem of $\mathrm{H}$. Wielandt [14].
\end{abstract}

\section{Introduction and the main results}

The classical Jordan-Hölder theorem for groups goes back to C. Jordan [8] and O. Hölder [7], see also the historical remark after Theorem 5.12 in J. J. Rotman [11]. R. Dedekind [2] was certainly aware (at least for the modular case) that the Jordan-Hölder theorem followed from the corresponding lattice theoretic statement. Our goal is to strengthen this theorem, both for groups and lattices, by adding a statement on uniqueness to it. Although we formulate the Jordan-Hölder theorem in a strong but somewhat technical form, which is due to G. Grätzer and J. B. Nation [6], this form (see the first part of Theorem 1 below) can easily be extracted from the classical proofs.

As usual, the relation "subnormal subgroup" is the transitive closure of "normal subgroup". For subnormal subgroups $A \triangleleft B$ and $C \triangleleft D$ of a given group $G$, the factor $B / A$ will be called subnormally down-and-up projective to $D / C$, if there are subnormal subgroups $X \triangleleft Y$ of $G$ such that $A Y=B$, $A \cap Y=X, C Y=D$ and $C \cap Y=X$. Clearly, $B / A \cong D / C$ in this case, because both are isomorphic with $Y / X$ by the Second Isomorphism Theorem.

Theorem 1. Let $\{1\}=N_{0} \triangleleft N_{1} \triangleleft \cdots \triangleleft N_{n}=G$ and $\{1\}=M_{0} \triangleleft M_{1} \triangleleft \cdots \triangleleft M_{m}=G$ be two composition series of a group $G$. Then

2000 Mathematics Subject Classification: 06C10 and 20D35.

Key words and phrases: Jordan-Hölder theorem, semimodular lattice, slim lattice, planar lattice, group, composition series, subnormal subgroup, chief series.

This research was supported by the NFSR of Hungary (OTKA), grant no. K77432. 
- $n=m$, and there exists a permutation $\pi$ of the set $\{1, \ldots, n\}$ such that $N_{i} / N_{i-1}$ is subnormally down-and-up projective to $M_{\pi(i)} / M_{\pi(i)-1}$ for all $i$;

- moreover, this permutation $\pi$ is uniquely determined, and it has the following property: if $i, j \in\{1, \ldots, n\}$ and $N_{i} / N_{i-1}$ is subnormally downand-up projective to $M_{j} / M_{j-1}$, then $j \geq \pi(i)$.

A maximal chain of normal subgroups is called a chief series. Stipulating that $X$ and $Y$ above are normal subgroups rather than subnormal ones, we obtain the definition of normal down-and-up projectivity.

Theorem 2. The same as Theorem 1 but "composition series" and "subnormally" are replaced by "chief series" and "normally" everywhere.

Both theorems will easily follow from their lattice theoretical counterpart. Indeed, if $G$ is a group with a finite composition series, then its subnormal subgroups form a sublattice $\mathfrak{H}(G)$ of the lattice of all subgroups by a classical result of H. Wielandt [14]; see also Theorem 1.1.5 and the remark after its proof in R. Schmidt [12], or see page 302 in M. Stern [13]. It is not hard to see that $\mathfrak{H}(G)$ is dually semimodular; see Theorem 2.1.8 in [12], or the proof of Theorem 8.3.3 in [13], or the proof of Theorem 9.8 in J. B. Nation [10]. Since we are going to formulate the lattice theoretical Jordan-Hölder theorem for semimodular lattices, as usual, "down-and-up" and " $j \geq \pi(i)$ " from Theorems 1 and 2 will, of course, be dualized.

Except for a short proof at the very end, the rest of the paper is purely lattice theoretical. Basic familiarity with lattices is assumed; however, only a very small part of, say, G. Grätzer [4] or J. B Nation [10] will be needed. For intervals $\left[a_{1}, b_{1}\right]$ and $\left[a_{2}, b_{2}\right]$ of a lattice, we say that $\left[a_{1}, b_{1}\right]$ is up-perspective to $\left[a_{2}, b_{2}\right]$, in notation $\left[a_{1}, b_{1}\right] \nearrow\left[a_{2}, b_{2}\right]$, if $a_{2} \vee b_{1}=b_{2}$ and $a_{2} \wedge b_{1}=a_{1}$. Dually, $\left[a_{2}, b_{2}\right] \searrow\left[a_{1}, b_{1}\right]$ means $\left[a_{1}, b_{1}\right] \nearrow\left[a_{2}, b_{2}\right]$. We say that $\left[a_{1}, b_{1}\right]$ is up-and-down projective to $\left[a_{2}, b_{2}\right]$, in notation $\left[a_{1}, b_{1}\right] \bigwedge\left[a_{2}, b_{2}\right]$, if there is an interval $[x, y]$ such that $\left[a_{1}, b_{1}\right] \nearrow[x, y]$ and $[x, y] \searrow\left[a_{2}, b_{2}\right]$. A lattice $L$ is called (upper) semimodular, if $b \vee c$ covers or equals $a \vee c$ for all for all $a, b, c \in L$ with $a \prec b$.

Theorem 3 (Main theorem). Assume that $C=\left\{0=c_{0} \prec c_{1} \prec \cdots \prec c_{n}=1\right\}$ and $D=\left\{0=c_{0} \prec c_{1} \prec \cdots \prec c_{m}=1\right\}$ are maximal chains of a semimodular lattice $L$. Then

- $n=m$, and there is a permutation $\pi$ of the set $\{1, \ldots, n\}$ such that the interval $\left[c_{i-1}, c_{i}\right]$ is up-and-down projective to the interval $\left[d_{\pi(i)-1}, d_{\pi(i)}\right]$, for all $i$;

- moreover, this permutation $\pi$ is uniquely determined, and it has the following property: if $i, j \in\{1, \ldots, n\}$ and $\left[c_{i-1}, c_{i}\right] \bigwedge\left[d_{j-1}, d_{j}\right]$, then $j \leq \pi(i)$.

The first part of Theorem 3 is due to G. Grätzer and J. B. Nation [6]; our contribution is the second part. In view of [6], one can say that semimodular 
lattices provide the foundational reason of the Jordan-Hölder theorem. Surprisingly, it will appear that the main role is played by planar semimodular lattices. Since these easy-to-visualize lattices and their properties are anyhow needed, we devote two lines, the proof of Corollary 16, to an entirely new approach to the first part of Theorem 3 .

One may ask if $j<\pi(i)$ can happen, or if we have uniqueness for projectivities. (By projectivity we mean the transitive closure of the up-and-down projectivity.) The answer is given by the following example.

Remark 4. Consider $M_{3}$, the five-element modular non-distributive lattice, which is the lattice of (normal) subgroups of the Klein four-group $\mathbb{Z}_{2} \times \mathbb{Z}_{2}$. Let $C$ and $D$ be maximal chains in $M_{3}$ such that $C \cap D=\{0,1\}$. Then $\pi(1)=2$ but $\left[c_{0}, c_{1}\right] \bigwedge\left[d_{j-1}, d_{j}\right]$ for $j=1,2$. Moreover, for both permutations $\tau$ of $\{1,2\},\left[c_{i-1}, c_{i}\right]$ is projective to $\left[c_{\tau(i)-1}, c_{\tau(i)}\right]$, for all $i$.

\section{Lemmas, proofs, and auxiliary lattices}

A finite lattice $M$ is called planar, if it has a planar diagram, that is a diagram in which the edges are non-horizontal straight lines that may intersect only at their endpoints. A planar lattice is finite by definition. Although always a fixed planar diagram is kept in mind, our statements will be valid no matter which planar diagram is considered. The edges of the (fixed) planar diagram divide the plane into regions; the minimal regions are called cells. The notion of cells are exemplified by the five-element non-distributive lattices: $N_{5}$ has only one cell while $M_{3}$ has two. Note that $M$ has no cell iff it is a chain. $M$ is said to be a 4-cell lattice, if it is planar and each cell is surrounded by exactly four edges. Then for each cell there are $a, b \in M$, called the left corner and the right corner of the cell, such that the cell is surrounded by the edges $a \wedge b \prec a$, $a \wedge b \prec b, a \prec a \vee b$ and $b \prec a \vee b$, and $a$ is on the left of $b$. The elements $a \wedge b$ and $a \vee b$ are called the bottom and the top of the cell, respectively. The left boundary and the right boundary of $M$ are denoted by $\mathcal{B}_{\text {left }}(M)$ and $\mathcal{B}_{\text {right }}(M)$, respectively. Although their meaning should be clear, we mention that a rigorous technical definition is given by D. Kelly and I. Rival [9]. Note that $\mathcal{B}_{\text {left }}(M)$ and $\mathcal{B}_{\text {right }}(M)$ are maximal chains in $M$. By a covering square we mean a subset $\{a \wedge b, a, b, a \vee b\}$ such that $a \wedge b \prec a, a \wedge b \prec b, a \prec a \vee b$ and $b \prec a \vee b$. For $a \in M$, the principal ideal $[0, a]=\{x \in M: x \leq a\}$ and the principal filter $[a, 1]$ will be denoted by $\downarrow a$ and $\uparrow a$, respectively.

By a slim lattice we mean a finite lattice $M$ such that $J(M)$, the poset of non-zero join-irreducible elements, contains no three-element antichain. In virtue of Dilworth [3], a finite lattice $M$ is slim iff $J(M)$ is the union of two chains. Lemma 6 will guarantee that slim lattices are planar but we now have to assume planarity in the second part of the following lemma. 


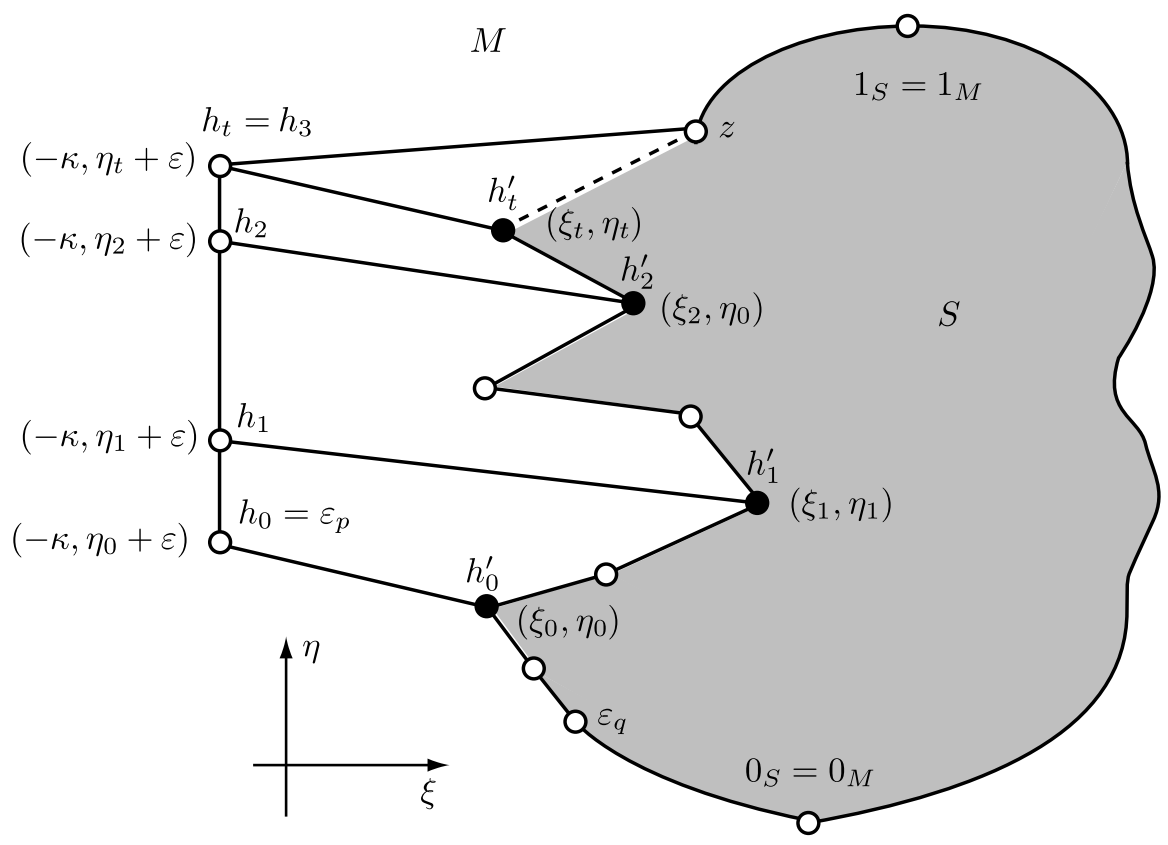

FiguRe 1. A planar extension of $S$ to $M$

Lemma 5. Let $M$ be a slim lattice. If $e$ is a maximal element of $J(M)$, then $\uparrow e$ is a chain. If, in addition, $M$ is a planar lattice (with a fixed planar diagram) and $e$ is on the left boundary of $M$, then $\uparrow e \subseteq \mathcal{B}_{\text {left }}(M)$.

Proof. Assume that $e$ is a maximal element of $J(L)$. Let $J(M)=U \cup V$ where $U$ and $V$ are chains. Then, say, $e \in U$. Each $x \in \uparrow e$ is of the form $x=u \vee v$ for some $u \in U$ and $v \in V$. However, then $x=e \vee x=e \vee u \vee v=e \vee v$, because $e$ is the largest element of the chain $U$. So $x=e \vee v$, and any other $x^{\prime} \in \uparrow e$ is $e \vee v^{\prime}$ for some $v^{\prime} \in V$. Since $V$ is a chain, $v$ and $v^{\prime}$ are comparable, whence so are $x$ and $x^{\prime}$. This shows that $\uparrow e$ is a chain.

Assume that, in addition, $e \in \mathcal{B}_{\text {left }}(M)$. For every $b \in \mathcal{B}_{\text {left }}(M)$ and $c \in \uparrow e$, either $e \leq b$ or $b \leq e$, since $\mathcal{B}_{\text {left }}(M)$ is a chain. The first possibility implies that $b$ and $c$ are comparable since $\uparrow e$ is a chain, while the second possibility implies the same trivially. Therefore $\mathcal{B}_{\text {left }}(M) \cup\{c\}$ is a chain. Since $\mathcal{B}_{\text {left }}(M)$ is a maximal chain, we get that $c \in \mathcal{B}_{\text {left }}(M)$, proving $\uparrow e \subseteq \mathcal{B}_{\text {left }}(M)$.

Lemma 6. Let $E=\left\{0=e_{0} \prec e_{1} \prec \cdots \prec e_{n}\right\}$ and $F=\left\{0=f_{0} \prec f_{1} \prec \cdots \prec\right.$ $\left.f_{m}\right\}$ be non-empty chains of a finite lattice $M$ such that $J(M) \subseteq E \cup F$. Then $M$ has a planar diagram such that $\mathcal{B}_{\text {left }}(M)=E \cup \uparrow e_{n}$ and $\mathcal{B}_{\text {right }}(M)=F \cup \uparrow f_{m}$.

Proof. We prove the lemma by induction on $|M|$. We assume that $|M| \geq 3$, $n \geq 1$ and $m \geq 1$, since otherwise the statement is trivial. Let $\max (J(L))$ denote the set of maximal join-irreducible elements, note that $|\max (J(M))| \leq$ 
2. Since at least one of $E$ and $F$ contains a maximal element of $J(M)$, we can assume that $e_{p} \in \max (J(M))$ for some $0<p \leq n$. Let $e_{q}$ and $f_{r}$ be the largest element of $\left\{e_{1}, \ldots, e_{p-1}\right\} \cap J(M)$ and $\left(F \backslash\left\{e_{p}\right\}\right) \cap J(M)$, respectively, and define $E^{\prime}:=\left\{0=e_{0} \prec e_{1} \prec \cdots \prec e_{q}\right\}$ and $F^{\prime}:=\left\{0=f_{0} \prec f_{1} \prec \cdots \prec f_{r}\right\}$. Denote by $S$ the join-subsemilattice generated by $E^{\prime} \cup F^{\prime}$, and let $H=M \backslash S$. Since $e_{p}$ is join-irreducible, $e_{p} \in H$. Clearly, $M \backslash \uparrow e_{p} \subseteq S$, that is, $H \subseteq \uparrow e_{p}$. We know from Lemma 5 that $\uparrow e_{p}$ is a chain. Consequently, its elements are $\wedge$-irreducible in $M$. Therefore $H$ is also a chain and its elements are $\wedge$-irreducible. This yields that $S$ is closed with respect to meet, that is, $S$ is a sublattice of $M$.

By the induction hypothesis, $S$ has a planar diagram such that

$$
\mathcal{B}_{\text {left }}(S)=E^{\prime} \cup\left[e_{q}, 1_{S}\right] \text { and } \mathcal{B}_{\text {right }}(S)=F^{\prime} \cup\left[f_{r}, 1_{S}\right] .
$$

For $x \in H$, if $f_{i}$ is the largest element of $\downarrow x \cap F^{\prime}$, then $x=e_{p} \vee f_{i}$, no matter if $e_{p}$ is in $F$ or not. So, $x^{\prime}:=e_{q} \vee f_{i}$ is the largest element of $\downarrow x \cap S$. Consider the mapping $\varphi: H \rightarrow S, x \mapsto x^{\prime}$. Since $x^{\prime}=\varphi(x) \in\left[e_{q}, 1_{S}\right], \varphi$ maps $H$ into $\mathcal{B}_{\text {left }}(S)$. Clearly, $\varphi$ is order-preserving, and it is injective since $x=e_{p} \vee x^{\prime}$.

Observe that the chain $H$ is a cover-preserving sublattice of $M$. Indeed, otherwise we would have $e_{p} \leq x_{1}<x_{2}<x_{3}$ with $x_{1}, x_{3} \in H$ but $x_{2} \in S$. Then $x_{3}=e_{p} \vee y_{3}$ for some $y_{3} \in F^{\prime}$ would imply $x_{3}=x_{2} \vee x_{3}=x_{2} \vee e_{p} \vee y_{3}=$ $x_{2} \vee y_{3} \in S$, a contradiction.

So, we can assume that $H=\left\{e_{p}=h_{0} \prec h_{1} \prec \cdots \prec h_{t}\right\}$ where $t \in \mathbb{N}_{0}$ and the covering is understood in $M$. For $i=0, \ldots, t$, let $h_{i}^{\prime}=\varphi\left(h_{i}\right)$ be the point $\left(\xi_{i}, \eta_{i}\right)$ of the plane (in the fixed planar diagram of $S$ ). The $h_{i}^{\prime}$ are the black-filled elements in Figure 1, where $t=3$. Since $h_{0}^{\prime}<h_{1}^{\prime}<\cdots<h_{t}^{\prime}$, we have $\eta_{0}<h_{1}<\cdots<\eta_{t}$. We have to distinguish two cases.

First, we assume that $h_{t} \neq 1_{M}$. Lemma 5 and $h_{t} \in \uparrow e_{p}$ yield that $h_{t}$ has a unique cover $z \in M$. Clearly, $z \in S$ and $1_{M}=1_{S}$. The dotted line in Figure 1 represents $\left[h_{t}^{\prime}, z\right]$. From $(1)$ and $h_{t}^{\prime} \in \uparrow e_{q}$ we obtain that $\left[h_{t}^{\prime}, z\right]$ is a chain in $S$, and $\left[h_{t}^{\prime}, z\right]$ and all the $h_{i}^{\prime}$ are on the left boundary of $S$. In the particular case when $\left[h_{t}^{\prime}, z\right]$ is two-element, the dotted line is an edge of $S$ that should be deleted since $h_{t}^{\prime}<h_{t}<z$ in $M$. Clearly, if $\varepsilon$ is a sufficiently small a positive number and $\kappa$ is large enough, then positioning $h_{i}$ to the point $\left(-\kappa, \eta_{i}+\varepsilon\right)$ keeps the planarity of the diagram, see Figure 1 . This way we get a planar diagram of $M$.

Next, we assume that $h_{t}=1_{M}$. Then $h_{t}^{\prime}=1_{S}$ and $z$ is not present. However, we get a planar diagram of $M$ in the same way as in the previous case.

Clearly, $\left\{e_{0}, \ldots, e_{p}\right\} \subseteq \mathcal{B}_{\text {left }}(M)$ and $\left\{f_{0}, \ldots, f_{r}\right\} \subseteq \mathcal{B}_{\text {right }}(M)$ in the planar diagram just obtained. So the statement follows from Lemma 5.

Lemma 7. For every finite lattice $M$, the following four conditions are equivalent:

- $M$ is a slim semimodular lattice;

- $M$ is a slim semimodular lattice and it is a planar 4-cell lattice; 
- $M$ is a planar semimodular lattice without cover-preserving $M_{3}$-sublattices;

- $M$ is a planar semimodular lattice in which 4-cells and covering squares are the same.

The third condition is clearly equivalent with the definition of G. Grätzer and E. Knapp [5]. This fact justifies a later reference to [5].

Proof. Clearly, the last two conditions are equivalent. The second condition trivially implies the first one.

Assume the first condition. Then $M$ is planar by Lemma 6 . If it contained a cover-preserving $M_{3}$-sublattice, then we could find three distinct covers $v_{1}, v_{2}, v_{3}$ of some $u \in M$ and $p_{i} \in\left(J(M) \cap \downarrow v_{i}\right) \backslash \downarrow u$ for $i \in\{1,2,3\}$, and $v_{i}=u \vee p_{i}$ would yield that $\left\{p_{1}, p_{2}, p_{3}\right\}$ is a three-element antichain in $J(L)$. Hence the third condition follows.

Assume the third condition. Then semimodularity implies that each cell of $M$ is a 4-cell, and Corollary 2 in [1] (in particular, the first sentence of its proof) gives that $J(L)$ has no three-element antichain. That is, the second condition holds.

In what follows, the notation of Theorem 3 will be fixed. In particular, $L$ is a semimodular lattice. Let $K=(K ; \vee, 0)$ be the subsemilattice of $(L ; \vee, 0)$ generated by $C \cup D$. Note that $K=(K ; \leq)$ is a lattice, but this auxiliary lattice is not a sublattice of $L$ in general. However, with an appropriate choice of planar representation, we have

Lemma 8. $K$, considered as a lattice, is a slim semimodular lattice with left boundary chain $C$ and right boundary chain $D$. Further, $(K ; \vee, 0)$ is a coverpreserving subsemilattice of $(L ; \vee, 0)$.

Proof. Clearly, $J(K) \subseteq C \cup D$. Hence $K$ is slim. Let $x \prec y$ in $K$, and let $s=\max \left\{i: c_{i} \leq x\right\}$ and $t=\max \left\{j: d_{j} \leq x\right\}$. Then $x=c_{s} \vee d_{t}$, and either $y=c_{s+1} \vee d_{t}$ or $y=c_{s} \vee d_{t+1}$. In both cases, the semimodularity of $L$ implies that $x \prec y$ in $L$. So, $K$ is a cover-preserving join-subsemilattice of $L$, whence $K$ is semimodular. Finally, we apply Lemma 6 .

The following lemma is a part of Lemma 4 of [5]. It also follows from the fact, included in the above proof, that each $x \in K$ has at most two covers.

Lemma 9 (G. Grätzer and E. Knapp [5]). No two distinct 4-cells of $K$ have the same bottom.

By a prime interval we mean a two-element interval $[a, b]$, that is, an interval $[a, b]$ with $a \prec b$. The set of all intervals and that of all prime intervals of a lattice $M$ are denoted by $\operatorname{Int}(M)$ and $\operatorname{Prin}(M)$, respectively. As usual, the projectivity relation on $\operatorname{Int}(M)$ is the transitive closure of " $\nearrow$ " $\cup$ " Projectivity should not be confused with the following notion. For $\left[a_{0}, a_{1}\right]$ and $\left[b_{0}, b_{1}\right]$ in $\operatorname{Prin}(M)$, we say that $\left[a_{0}, a_{1}\right]$ is $\operatorname{Prin}(M)$-projective to $\left[b_{0}, b_{1}\right]$, 


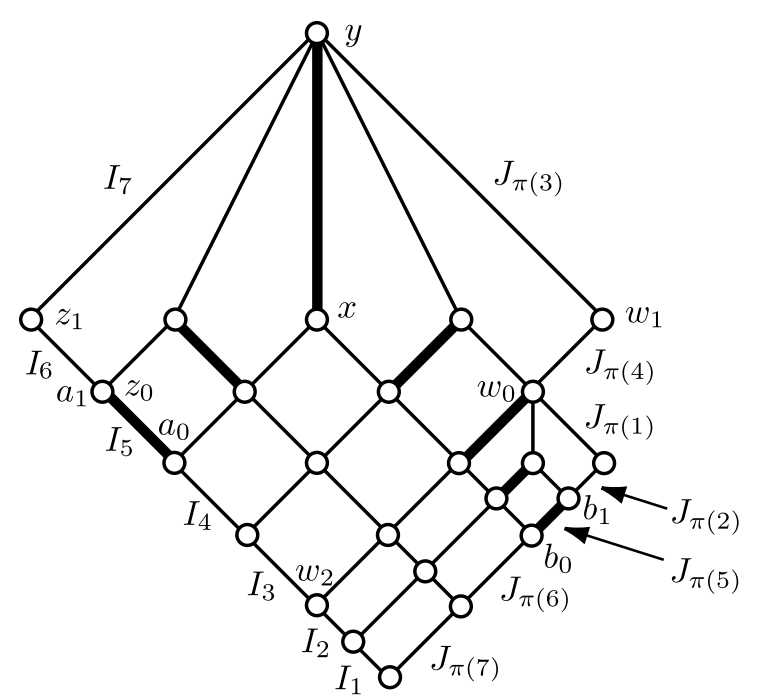

Figure 2. A slim semimodular lattice

if there are $k \in \mathbb{N}_{0}$ and $\left[x_{i}, y_{i}\right] \in \operatorname{Prin}(M)$ for $i \leq k$ such that $\left[a_{0}, a_{1}\right]=$ $\left[x_{0}, y_{0}\right],\left[b_{0}, b_{1}\right]=\left[x_{k}, y_{k}\right]$, and, for all $i \in\{1, \ldots, k\},\left[x_{i-1}, y_{i-1}\right] \nearrow\left[x_{i}, y_{i}\right]$ or $\left[x_{i-1}, y_{i-1}\right] \searrow\left[x_{i}, y_{i}\right]$. One may ask if $\operatorname{Prin}(M)$-projectivity coincides with projectivity at least in particular cases. The answer is given below.

Remark 10. Let $M$ be the principal filter $\uparrow w_{2}$ in Figure 2. Then $M$ is a slim semimodular lattice. The prime intervals $\left[z_{0}, z_{1}\right]$ and $\left[w_{0}, w_{1}\right]$ are on its left boundary and right boundary, respectively. These intervals are projective in $M$, but they are not $\operatorname{Prin}(M)$-projective. Note also that $\left[z_{0}, z_{1}\right]$ and $\left[w_{0}, w_{1}\right]$ are projective via ten perspectivity steps but not in fewer steps.

Lemma 11. Let $M$ be a semimodular lattice of finite length, and let $\left[a_{0}, a_{1}\right]$, $\left[b_{0}, b_{1}\right] \in \operatorname{Prin}(M)$. Then these two prime intervals are $\operatorname{Prin}(M)$-projective iff there are $k \in \mathbb{N}_{0}$ and $\left[x_{i}, y_{i}\right] \in \operatorname{Prin}(M)$ for $i \leq k$ such that $\left[a_{0}, a_{1}\right]=\left[x_{0}, y_{0}\right]$, $\left[b_{0}, b_{1}\right]=\left[x_{k}, y_{k}\right]$, and, for all $i \in\{1, \ldots, k\},\left\{x_{i-1}, y_{i-1}, x_{i}, y_{i}\right\}$ is a covering square.

Proof. Assume that $[a, b],[c, d] \in \operatorname{Prin}(M)$ such that $[a, b] \nearrow[c, d]$. Take a chain $a=z_{0} \prec z_{1} \prec \cdots \prec z_{t}=b$, and define $z_{i}^{\prime}=z_{i} \vee b$. Then $\left\{z_{i-1}, z_{i}, z_{i-1}^{\prime}, z_{i}^{\prime}\right\}$ is a covering square by semimodularity. If $[a, b] \searrow[c, d]$, then $[c, d] \nearrow[a, b]$, and we obtain covering squares similarly. So, each perspectivity step gives rise to some covering squares, and the collection of all these squares prove the "only if" part. The "if" part is evident.

Clearly, $\operatorname{Prin}(M)$-projectivity is an equivalence relation on $\operatorname{Prin}(M)$. In $K$, defined right before Lemma 8, the blocks (in other word, classes) of Prin $(K)$ projectivity will be called trajectories. Let us emphasize that trajectories, 
unless otherwise stated, are defined and will be used only for the lattice $K$. The terminology is explained by the following lemma (and its proof).

Lemma 12. The trajectories of $K$ start at the left boundary chain $C$. First they go upwards (possibly in zero step), then they go downwards (possibly in zero step), and finally they reach the right boundary chain D. Trajectories never ramify.

Proof. Take a prime interval $[x, y]$ in a trajectory $T$. By planarity, $[x, y]$ is the side of at most two adjacent covering squares. Indeed, it is on the left boundary of at most one square, and it is on the right boundary of at most one square. The opposite sides of these squares also belong to $T$. Repeating the same argument to these opposite sides and continuing to the left and to the right, we can see by Lemma 11 that $T$ is a sequence of prime intervals such that any two consecutive prime intervals form a covering square. For example, a trajectory of the slim semimodular lattice depicted in Figure 2 is indicated by thick lines.

We can think of $T$ as a sequence of prime intervals that "goes" from the left boundary $C$ to the right boundary $D$. Since no edge is on the left boundary of two different covering squares, $T$ cannot ramify while going to the right. However, while going from the left to the right, (segments of) $T$ can go upwards (that is, to the northeast) or downwards (to the southwest). For example, the section from $\left[a_{0}, a_{1}\right]$ to $[x, y]$ of the trajectory in Figure 2 goes upwards, while the section from $[x, y]$ to $\left[b_{0}, b_{1}\right]$ goes downwards. Notice that a trajectory can turn down only where two distinct 4-cells have the same top.

A downward going section of $T$ cannot be followed by an upward going section. Indeed, a down-going section could turn upwards only where two distinct 4-cells would have the same bottom, but this is impossible in virtue of Lemma 9 .

Lemma 13. For any two prime intervals of $K$, these intervals are up-anddown projective iff they belong to the same trajectory.

Proof. Suppose that $\left[a_{0}, a_{1}\right],\left[b_{0}, b_{1}\right] \in \operatorname{Prin}(K)$ are up-and-down projective. Then $\left[a_{0}, a_{1}\right] \nearrow[x, y] \searrow\left[b_{0}, b_{1}\right]$ for some $[x, y] \in \operatorname{Int}(K)$. Since $x \prec y$ by semimodularity, $\left[a_{0}, a_{1}\right]$ and $\left[b_{0}, b_{1}\right]$ are $\operatorname{Prin}(K)$-projective, whence they belong to the same trajectory.

Conversely, assume that $\left[a_{0}, a_{1}\right]$ and $\left[b_{0}, b_{1}\right]$ are prime intervals belonging to the same trajectory $T$. By Lemma 12 , the section of $T$ between $\left[a_{0}, a_{1}\right]$ and $\left[b_{0}, b_{1}\right]$ first goes upwards (possibly in zero step), and then it goes downwards (possibly in zero step). Since the composite of up-perspectivities is an up-perspectivity, and the same holds for down-perspectivities, it follows that $\left[a_{0}, a_{1}\right] \bigwedge\left[b_{0}, b_{1}\right]$.

Lemma 14. If $M$ is lattice, $\left[a_{0}, a_{1}\right],\left[b_{0}, b_{1}\right] \in \operatorname{Int}(M)$ and $\left[a_{0}, a_{1}\right] \bigwedge\left[b_{0}, b_{1}\right]$, then $a_{1} \not \leq a_{0} \vee b_{0}$ and $b_{1} \not \leq a_{0} \vee b_{0}$. 


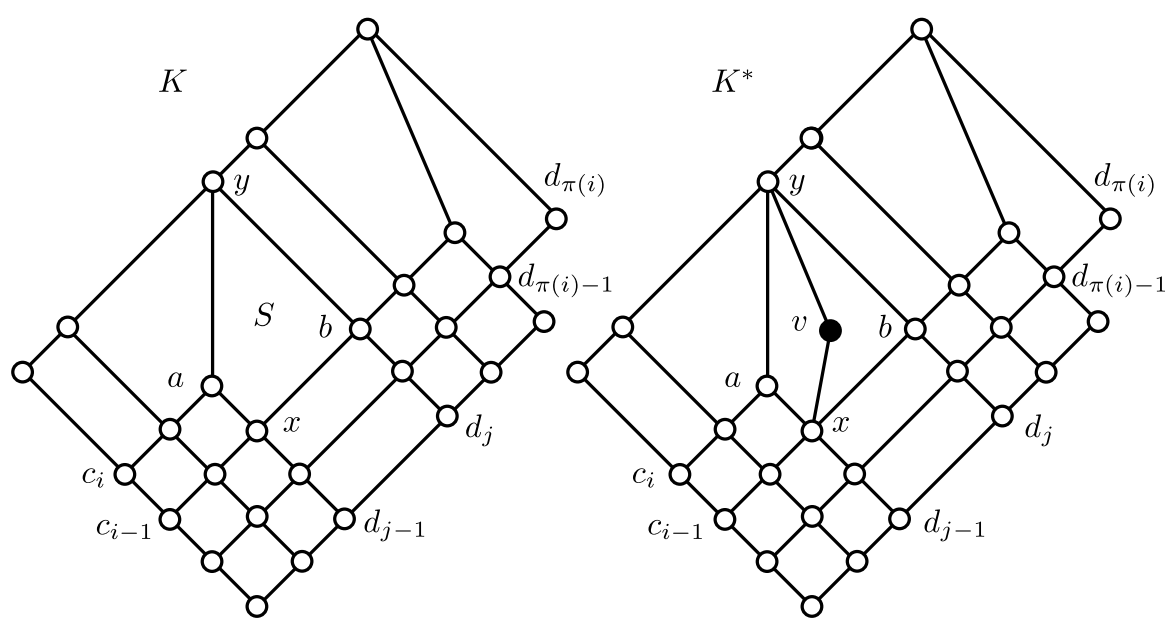

Figure $3 . K$ and $K^{*}$

Proof. Choose an $[x, y] \in \operatorname{Int}(M)$ with $\left[a_{0}, a_{1}\right] \nearrow[x, y] \searrow\left[b_{0}, b_{1}\right]$. Then $a_{0} \vee$ $b_{0} \leq x$. If, say, $a_{1} \leq a_{0} \vee b_{0}$, then $y=x \vee a_{1}=x$, a contradiction.

Lemma 15. The correspondence $\{(i, \ell): 1 \leq i \leq n, 1 \leq \ell \leq m$ and $\left[c_{i-1}, c_{i}\right] \bigwedge\left[d_{\ell-1}, d_{\ell}\right]$ in $\left.K\right\}$ is a bijection $\pi:\{1, \ldots, n\} \rightarrow\{1, \ldots, m\}$.

Proof. We obtain from Lemmas 12 and 13 that for each $i$ there is exactly one $\ell$ with $\left[c_{i-1}, c_{i}\right] \bigwedge\left[d_{\ell-1}, d_{\ell}\right]$. Left-right symmetry yields the converse.

Corollary 16. The (previously known) first part of Theorem 3 holds.

Proof. By Lemma 8, $\left[c_{i-1}, c_{i}\right] \bigwedge\left[d_{\ell-1}, d_{\ell}\right]$ in $K$ implies $\left[c_{i-1}, c_{i}\right] \bigwedge\left[d_{\ell-1}, d_{\ell}\right]$ in $L$. Thus, Lemma 15 applies.

The bijection $\pi$ defined in Lemma 15 will be called the planar matching. Remember that, for all $i \in\{1, \ldots, n\}$,

$$
\left[c_{i-1}, c_{i}\right] \bigwedge\left[d_{\pi(i)-1}, d_{\pi(i)}\right] \text { in } K \text { and also in } L,
$$

and these two prime intervals belong to the same trajectory of $K$. Note that once $K$ is depicted, the planar matching $\pi$ is very easy to find; see Figure 2, where $I_{i}$ and $J_{\pi(i)}$ denote $\left[c_{i-1}, c_{i}\right]$ and $\left[d_{\pi(i)-1}, d_{\pi(i}\right]$, respectively.

For the rest of the paper, let us fix a pair $(i, j) \in\{1, \ldots, n\}^{2}$ such that

$$
\left[c_{i-1}, c_{i}\right] \bigwedge\left[d_{j-1}, d_{j}\right] \text { holds in } L \text {. }
$$

Lemma 17. Let $x=c_{i-1} \vee d_{j-1}, y=c_{i} \vee d_{j}, a:=c_{i} \vee x$ and $b:=d_{j} \vee x$. If $j \neq \pi(i)$, then $S=\{x, a, b, y\}$ is a covering square in $K$ with left corner $a$ and right corner $b$.

Proof. Notice that $y=a \vee b$. Since $\left[c_{i-1}, c_{i}\right] \bigwedge\left[d_{j-1}, d_{j}\right]$ in $L$, Lemma 14 implies that $c_{i}, d_{j} \not \leq x$. Hence $x \prec a \leq y$ and $x \prec b \leq y$. Suppose that $a=b$. Then $\left[c_{i-1}, c_{i}\right] \nearrow[x, a]=[x, b] \searrow\left[d_{j-1}, d_{j}\right]$ and, therefore, $\left[c_{i-1}, c_{i}\right] \bigwedge$ 
$\left[d_{j-1}, d_{j}\right]$ in $K$. Since $j \neq \pi(i)$, this contradicts $(2)$ in virtue of Lemma 15 . Hence $a \neq b$, and $S$ is a covering square in $K$ by semimodularity.

Before the rigorously formulated proof of Theorem 3, we outline the idea loosely. If $\left.\left[c_{i-1}, c_{i}\right] \searrow_{j-1}, d_{j}\right]$ in $L$ and $j \neq \pi(i)$, then we insert a new element $v$ into the 4-cell $S$ of $K$ defined in Lemma 17 in order to obtain a lattice $K^{*}$ such that $\left[c_{i-1}, c_{i}\right] \searrow\left[d_{j-1}, d_{j}\right]$ already in $K^{*}$, see Figure 3 . There is a unique trajectory $T$ of $K$ that starts at $\left[c_{i-1}, c_{i}\right]$ and ends at $\left[d_{\pi(i)-1}, d_{\pi(i)}\right]$. The new element ramifies $T$ in $K^{*}$. The original part goes upwards after $S$ within $K$, it may turn downwards only later, and it stops at $\left[d_{\pi(i)-1}, d_{\pi(i)}\right]$. The new part turns downwards in $K^{*}$ immediately at $S$, then it keeps going downwards in $K$, and stops at $\left[d_{j-1}, d_{j}\right]$. This explains visually why $\left[d_{j-1}, d_{j}\right]$ is lower on the right boundary chain than $\left[d_{\pi(i)-1}, d_{\pi(i)}\right]$, that is, why $j \leq \pi(i)$.

Proof of Theorem 3. Corollary 16 settles the first part of Theorem 3. It suffices to show the stated " $\left[c_{i-1}, c_{i}\right] \bigwedge\left[d_{j-1}, d_{j}\right] \Rightarrow j \leq \pi(i)$ " property of the planar matching $\pi$, since it clearly implies the desired uniqueness. Hence, by way of contradiction, we assume (3) together with $\pi(i)<j$.

By Lemma $7, S$ defined in Lemma 17 is a 4 -cell in $K$. We add a doubly irreducible new element $v$ to the interior of $S$, see Figure 3. In other words, we change $S$ into a covering $M_{3}$ sublattice. This way we obtain a new lattice $K^{*}$.

Clearly, $K^{*}$ is a 4-cell planar lattice. Trivially, or using Lemma 2 of G. Grätzer and E. Knapp [5], we get that that $K^{*}$ is semimodular. Notice that $K$ is a sublattice of $K^{*}$ but, in general, $\left(K^{*} ; \vee, 0\right)$ is not a subsemilattice of $(L ; \vee, 0)$. Since $\pi(i)<j$ gives that $d_{\pi(i)}<d_{j}$ and we know that $d_{j} \leq x \vee d_{j}=b$, we obtain $d_{\pi(i)-1}<d_{\pi(i)}<b$. Hence $d_{\pi(i)} \leq b=b \vee d_{\pi(i)-1}$, and Lemma 14 yields that

$[b, y]$ is $\underline{\text { not }}$ up-and-down projective to $\left[d_{\pi(i)-1}, d_{\pi(i)}\right]$ in $K$.

On the other hand, we know that $\left[c_{i-1}, c_{i}\right] \nearrow[x, a] \nearrow[b, y]$. This gives that $\left[c_{i-1}, c_{i}\right] \nearrow[b, y]$, implying $\left[c_{i-1}, c_{i}\right] \bigwedge[b, y]$ in $K$. Hence Lemma 13 yields that $[b, y]$ belongs to the unique trajectory $T$ (of $K$ ) that contains $\left[c_{i-1}, c_{i}\right]$. But $\left[d_{\pi(i)-1}, d_{\pi(i)}\right]$ also belongs to $T$ by $(2)$ and Lemma 13 . Thus, $[b, y]$ and $\left[d_{\pi(i)-1}, d_{\pi(i)}\right]$ belong to the same trajectory of $K$, whence Lemma 13 implies $\left.[b, y] d_{\pi(i)-1}, d_{\pi(i)}\right]$ in $K$, which contradicts $(4)$.

Proof of Theorems 1 and 2. Assume that $G$ is a group with a finite composition series. As mentioned right after Theorem 2, the subnormal subgroups of $G$ form a dually semimodular sublattice $\mathfrak{H}(G)$ of the lattice of all subgroups.

If $A, B \in \mathfrak{H}(G)$ and $A \subseteq B$, then $A \in \mathfrak{H}(B)$ by Lemma 1.1.4 of [12], see also the proof of Theorem 9.8 in [10]. Therefore the composition series of $G$ are exactly the maximal chains of $\mathfrak{H}(G)$. Hence Theorem 1 becomes a corollary of Theorem 3. So does Theorem 2, because the lattice of normal 
subgroups is well-known to be modular; see, for example, Theorem 2.1.4 in R. Schmidt [12].

Acknowledgment. The authors thank the referee for calling their attention to $[2]$.

\section{REFERENCES}

[1] Czédli, G., Schmidt, E. T.: How to derive finite semimodular lattices from distributive lattices? Acta Mathematica Hungarica, 121 (2008), 277-282.

[2] Dedekind, R.: Über die von drei Moduln erzeugte Dualgruppe. Math. Annalen 53 (1900), 371-403.

[3] Dilworth, R. P.: A decomposition theorem for partially ordered sets. Ann. of Math. 51 (1951), 161-166.

[4] Grätzer, G.: General Lattice Theory, 2nd edn. Birkhäuser Verlag, Basel (1998).

[5] Grätzer, G., Knapp, E.: Notes on planar semimodular lattices I. Construction. Acta Sci. Math. (Szeged), 73 (2007), 445-462.

[6] Grätzer, G., Nation, J. B.: A new look at the Jordan-Hölder theorem for semimodular lattices. Algebra Universalis, to appear; see http://www . maths . umanitoba.ca/homepages/gratzer.html

[7] Hölder, O.: Zurückführung einer beliebigen algebraischen Gleichung auf eine Kette von Gleichungen. Math. Ann., 34 (1889), 26-56.

[8] Jordan, C.: Traité des substitutions et des équations algebraique. Gauthier-Villars, 1870.

[9] Kelly, D., Rival, I.: Planar lattices. Canad. J. Math., 27 (1975), 636-665.

[10] Nation, J. B.: Notes on Lattice Theory. 1991, http://www. math.hawaii.edu/ ${ }^{j b / b o o k s . h t m l ~}$

[11] Rotman, J. J.: An Introduction to the Theory of Groups, 4th edn. Graduate Texts in Mathematics, 148. Springer-Verlag, 1995.

[12] Schmidt, R.: Subgroup Lattices of Groups, de Gruyter Expositions in Mathematics, 14. Walter de Gruyter \& Co., Berlin, 1994.

[13] Stern, M.: Semimodular Lattices. Theory and Applications, Encyclopedia of Mathematics and its Applications, 73. Cambridge University Press, 1999.

[14] Wielandt, H.: Eine Verallgemeinerung der invarianten Untergruppen. Math. Zeit. 45 (1939), 209-244.

GÁBor CZÉdLI

University of Szeged, Bolyai Institute, Szeged, Aradi vértanúk tere 1, HUNGARY 6720 e-mail: czedli@math.u-szeged.hu

URL: http://www.math.u-szeged.hu/ czedli/

E. TAmÁs SchmidT

Mathematical Institute of the Budapest University of Technology and Economics, Mủegyetem rkp. 3, H-1521 Budapest, Hungary

e-mail:schmidt@math.bme.hu

$U R L:$ http://www.math.bme.hu/ schmidt/ 